\title{
ON THE MINIMAL VOLUME HYPERBOLIC 3-ORBIFOLD
}

\author{
F. W. Gehring and G. J. Martin
}

In this note we announce new results concerning the minimal volume 3-dimensional hyperbolic orbifold. The work is based on our sequence of papers [GM1-5]. Details will appear in [GM6-7].

A hyperbolic 3 -orbifold $\mathcal{Q}$ is the orbit space of a Kleinian group $\Gamma$. That is

$$
\mathcal{Q}=\mathbf{H}^{3} / \Gamma
$$

where $\Gamma$ is a discrete nonelementary orientation preserving group of isometries of hyperbolic 3-space $\mathbf{H}^{3}$. $\mathcal{Q}$ is a hyperbolic $3-$ manifold if $\Gamma$ is torsion free. We are therefore concerned with the problem of determining the Kleinian group of minimal co-volume.

We refer the reader to $[\mathrm{B}],[\mathrm{M}]$ and $[\mathrm{T}]$ for the basic facts concerning Kleinian groups and hyperbolic geometry. Before stating the main result we first recount a little of what is known.

In the two dimensional case, that is for Fuchsian groups, it is well known that the $(2,3,7)$-triangle group is the unique group of minimal co-area. It is the index two subgroup of the group generated by reflections in the sides of a hyperbolic triangle with interior angles $\pi / 2, \pi / 3$ and $\pi / 7$. Therefore the area is $\pi / 21$. One readily deduces from this fact the famous $84(g-1)$ theorem of Hurwitz $[\mathrm{H}]$ concerning the order of the automorphism group of a closed Riemann surface (the orbit space of a co-compact torsion free Fuchsian group) in terms of its genus $g$. Indeed, from the Riemann-Hurwitz formula for Fuchsian groups (sometimes called the signature formula [B]), one obtains the complete spectrum of possible areas for hyperbolic 2-folds.

The situation in higher dimensions is much less understood. Kazdan and Margulis proved the existence of a lower bound for the volume of hyperbolic $n$-folds, $n \geq 3$, in their paper establishing Selberg's hypothesis [KM].

1991 Mathematics Subject Classification. Primary 30F40, 57M50; Secondary 20H10. Received October 1, 1993.

F. W. Gehring was supported in part by grants from the NSF.

G. J. Martin was supported in part by grants from the N.Z. Foundation for Research, Science and Technology. 
Jørgensen and Thurston showed that the set of hyperbolic 3-manifold volumes is well ordered [T]. Similarly, the set of hyperbolic 3-orbifold volumes is well ordered [A2]. There have been some special minimal volume hyperbolic 3 -folds identified. These include the minimal volume noncompact orbifold [Me], the minimal volume noncompact manifold [A1], the minimal volume arithmetic orbifold $[\mathrm{CF}]$, the minimal volume manifold with totally geodesic boundary $[\mathrm{KoM}]$, the minimal limit volume orbifold [A2] and the minimal volume orbifold with singular set of degree 6 [GM4].

The main results announced here are the following.

Theorem 0.1. The unique Kleinian group of minimal co-volume which contains a torsion element of order $p \geq 4$ is a $Z_{2}$-extension $\Gamma_{353}$ of the orientation preserving index 2 subgroup of the group generated by reflections in the sides of the hyperbolic tetrahedron with Coxeter diagram 3-5-3. In particular, $\Gamma_{353}$ is arithmetic and has co-volume

$$
\operatorname{Vol}_{h y p}\left(\mathbf{H}^{3} / \Gamma_{353}\right)=0.039050 \ldots
$$

The finite subgroups of a Kleinian group are either cyclic, dihedral or one of the symmetry groups of a regular polyhedron, that is, the tetrahedral, octahedral or icosahedral groups. The following deals with these last three possibilities.

Theorem 0.2. The unique Kleinian group of minimal co-volume which contains a tetrahedral, octahedral or icosahedral subgroup is the group $\Gamma_{353}$.

Thus, in order to completely determine the Kleinian group of minimal co-volume, there remains only the problem of establishing a lower bound, exceeding $0.039 \ldots$, for the co-volume of a Kleinian $\Gamma$ group satisfying the following hypothesis : $\Gamma$ is co-compact and every finite subgroup is either

(1) the trivial group $\{1\}$,

(2) the cyclic groups $C_{2}$, of order 2 , or $C_{3}$, of order 3 ,

(3) the dihedral groups $D_{2}$, of order 4 , or $D_{3}$, of order 6 .

We expect the minimal co-volume group satisfying this hypothesis to have significantly larger co-volume than $0.039 \ldots$. For instance when $\Gamma$ is torsion free and the orbifold is a manifold, the smallest known volume is about $0.91 \ldots$ [W]. Culler and Shalen have made substantial progress in obtaining realistic volume estimates in the manifold case under assumptions on the first Betti number [CS 1-2]. In particular they show that if the first Betti number is at least 2, then the volume exceeds 0.34. They also eliminate all but a few special cases when the Betti number is at least 1 . In addition, our current estimates show that there are only a very small number of conjugacy classes of the finite subgroups occuring in 2 and 3 
above. Indeed we are very close to eliminating the possibilities $C_{3}$ and $D_{3}$ entirely.

Thus one can view the remaining part of the problem as obtaining a volume bound for hyperbolic 3 -orbifolds with a singular set of very simple structure.

Our methods are based on the construction of various types of precisely invariant sets for Kleinian groups and then on an analysis of the stabilizers of such sets. A set $F$ is precisely invariant under $\Gamma$ if for each $g \in \Gamma$ either

$$
g(F) \cap F=\emptyset \quad \text { or } \quad g(F)=F .
$$

If $F$ is precisely invariant under $\Gamma$ and if $\Gamma_{F}$ denotes the stabilizer of $F$, then

$$
\operatorname{Vol}_{\text {hyp }}\left(\mathbf{H}^{3} / \Gamma\right) \geq \operatorname{Vol}_{\text {hyp }}\left(F / \Gamma_{F}\right) .
$$

The point here is that the stabilizer of $F$ is usually virtually abelian and therefore somewhat easier to analyze than $\Gamma$. For instance, if $F$ is a fundamental domain, then $\Gamma_{F}=\{1\}$ and we have equality in (4). A key feature of this idea is simply that the size of maximal precisely invariant sets comes in a discrete spectra. Our proof is based on consideration of the maximal order of torsion elements in the group $\Gamma$. Then in each instance one need carefully analyze only a small number of possible extremal examples before obtaining sufficient bounds for the general case.

\section{The main ingredients}

Here is a summary of the main ingredients and new tools used in the proof.

1.1. Iteration theory and discreteness. The connection between iteration theory and discreteness in Kleinian groups, announced in [GM1], is generalized in [GM5] to produce new inequalities for Kleinian groups with an elliptic generator. A disk covering technique, using the more general polynomial trace identities given in [GM5], is used to propagate these inequalities. We are therefore able to describe substantial portions of the space of two generator Kleinian groups when one generator is elliptic [GM8]. The space of all such groups, discrete or otherwise, is a two complex dimensional space. Various symmetries provide a projection onto a one dimensional slice. In particular, one can assume the other generator has order two. Then the one free parameter left is essentially the trace of the commutator of the generators $[f, g]=f g f^{-1} g^{-1}$. The possible commutator values come in a discrete part and a continuous part, the continuous part arising from the groups which are discrete and free on the two generators. The extremal cases lie in the discrete part of the commutator spectrum and there we identify in [GM8] all small commutator values. 
1.2. The geometry of commutators. Suppose that $\Gamma$ is a Kleinian group and $f, g \in \Gamma$ are elliptic. The axis of $f$, axis $(f)$, is the closed hyperbolic line joining the fixed points of $f$ in $\partial \mathbf{H}^{3}$. Let $\delta=\delta(f, g)$ denote the hyperbolic distance between axis $(f)$ and axis $(g)$, and $\theta=\theta(f, g)$ the dihedral angle between the two spheres containing the axis and the common perpendicular. Then

$$
\sinh ^{2}(\delta+\imath \theta)=\frac{4 \gamma(f, g)}{\beta(f) \beta(g)}
$$

where

$$
\gamma(f, g)=\operatorname{tr}[f, g]-2, \quad \beta(f)=\operatorname{tr}^{2}(f)-4, \quad \beta(g)=\operatorname{tr}^{2}(g)-4,
$$

[GM5]. Hence

$$
\cosh (2 \delta)=\left|\frac{4 \gamma(f, g)}{\beta(f) \beta(g)}+1\right|+\left|\frac{4 \gamma(f, g)}{\beta(f) \beta(g)}\right|
$$

For instance, if $\beta(f)=\beta(g)$ we find that bounding $\delta$ from below provides precisely invariant neighborhoods of elliptic axes since $\delta\left(h f h^{-1}, f\right)$ is the hyperbolic distance between $h(\operatorname{axis}(f))$ and axis $(f)$. Thus we must exclude elliptical regions from the possible commutator spectrum for discrete groups. The discreteness of the initial commutator spectrum also implies discreteness of the spectrum of axial distances. The smallest axial distances are given in [GM3] and [GM5]. However, for our application here, somewhat more information is needed in certain extremal cases [GM8].

1.3. Collaring theorems. We say that an elliptic $f \in \Gamma$ is simple if it has a precisely invariant axis. The classification of the elementary discrete groups implies that elliptics of order $p \geq 7$ are simple. If an elliptic is simple, we find there is a precisely invariant neighborhood of a definite size about this axis. Such a neighborhood is known as a precisely invariant collar. It is a solid hyperbolic cylinder. The boundary of this cylinder is a standard euclidean cylinder in the induced metric. The stabilizer of the collar acts as a group of euclidean isometries in this induced metric on the boundary of the collar. Studying the way such groups act on collars yields the following.

Theorem 1.1. Let $\Gamma$ be a Kleinian group and $\mathcal{C}$ a maximal precisely invariant collar of hyperbolic radius $\rho$. Then

$$
V_{\text {ol }}\left(\mathbf{H}^{3} / \Gamma\right) \geq V(\rho)
$$


where

$V(\rho)=\sinh ^{2}(\rho) \int_{0}^{a(\rho)} \arccos \left(\left(1+\frac{1}{4} \operatorname{csch}^{2}(\rho)\right) \frac{\cosh (x)}{\cosh (a(\rho))}-\frac{1}{4} \operatorname{csch}^{2}(\rho)\right) d x$

and

$$
a(\rho)=\operatorname{arcsinh}(\sinh (\rho) / \cosh (2 \rho)) .
$$

A point to notice in Theorem 1.1 is that the assumed maximality of the collar $\mathcal{C}$ implies a lower bound on the translation length of any loxodromic fixing $\mathcal{C}$. This is because the shorter the translation length of the loxodromic, the larger the radius of the precisely invariant collar.

The bound in Theorem 1.1 is good for moderate collar radii. In other cases, when there is the possibility that the stabilizer of a collar contains a loxodromic with short translation length, we utilize another sharp collaring theorem which leads to the following result.

Theorem 1.2. Let $\Gamma$ be a Kleinian group and $f \in \Gamma$ a simple elliptic of order $p \geq 2$. Suppose that $\operatorname{Vol}_{\text {hyp }}\left(\mathbf{H}^{3} / \Gamma\right)<\infty$. Then there is a loxodromic $h \in \Gamma$ with axis $(h)=\operatorname{axis}(f)$ and translation length $\tau>0$ such that

$$
V \operatorname{ol}_{h y p}\left(\mathbf{H}^{3} / \Gamma\right) \geq \frac{\pi \tau}{2 p}\left(\frac{\sqrt{1-s_{p}(\tau)}}{s_{p}(\tau)}-\frac{1}{2}\right),
$$

where

$$
s_{p}(\tau)=\min _{j \geq 1} \min _{k \in \mathbb{Z}} \max _{0 \leq \theta<\pi} 2\left(\cosh (j \tau)-\cos \left(j \theta+\frac{2 k \pi}{p}\right)\right)
$$

The estimate

$$
s_{p}(\tau) \leq 2 \cosh \left(\sqrt{\frac{4 \pi \tau}{p \sqrt{3}}}\right)-2,
$$

arising from a diophantine analysis, shows that the volume estimate is good for small $\tau$. The estimate is automatically good for large $\tau$ because of the existence of precisely invariant collars of a fixed size.

1.4. The finite spherical triangle groups. It may well be the case that there is no simple elliptic axis. Indeed this is so in the extremal situation. Since we are assuming that there is torsion, we conclude $\Gamma$ contains either a parabolic transformation or a finite spherical triangle group. Since the former case has been dealt with in $[\mathrm{Me}]$ and since dihedral and cyclic groups have precisely invariant axes, we may assume the finite subgroup is the icosahedral, octahedral or tetrahedral group. We say that a point $P \in \mathbf{H}^{3}$ 
is an icosahedral, octahedral or tetrahedral point of $\Gamma$ if $P$ is stabilized by an iscosahedral, octahedral or tetrahedral subgroup of $\Gamma$, respectively.

In $[\mathrm{DM}]$, Derevnin and Mednykh show by an elegant geometric argument that the minimal distance between icosahedral points of a discrete group is surprisingly large and that the extremal case is realized when the points lie on a common axis of order 5. Their argument fails for octahedral and tetrahedral points and is not quite sufficient for our purposes. However, using different methods, we are able to identify the next icosahedral distance, the corresponding distances for the octahedral and tetrahedral points and some extreme cases. This requires close examination of the commutator spectrum for Kleinian groups generated by two (conjugate) spherical triangle groups. Then given the existence of an icosahedral, octahedral or tetrahedral point $\mathrm{P}$, we conclude the existence of a precisely invariant ball centered at $\mathrm{P}$ of radius half the corresponding minimal distance between such points.

\section{A sketch of the proof}

We first induct downwards on the maximal order $p \geq 4$ of torsion in the Kleinian group $\Gamma$.

2.1. The case $p \geq 6$. In [GM4] we identified the Kleinian group of minimal co-volume containing torsion of order 6 as $\Gamma(6)=P G L(2, O(\sqrt{-3}))$. Knowledge of the spectra of axial distance for $p \geq 7$ together with Theorem 1.1 implies that the co-volume of this group is exceeded by the co-volume of any Kleinian group containing an elliptic of order $p \geq 9$. In the extremal case for $p=7,8$ there is a $(2,3, p)$-triangle subgroup. The hyperplane stabilized by this subgroup is precisely invariant. A geometric argument in these last two cases yields the following.

Theorem 2.1. Let $\Gamma(p)$ be a smallest co-volume Kleinian group containing an elliptic of order $p \geq 6$. Then

$$
\operatorname{Vol}_{h y p}\left(\mathbf{H}^{3} / \Gamma(p)\right) \geq \operatorname{Vol}_{h y p}\left(\mathbf{H}^{3} / \Gamma(6)\right)=0.0845 \ldots
$$

with equality only when $p=6$.

Conjectured extremals for the case where $p \geq 7$ have been constructed in $[\mathrm{CM}]$. They are the index 2 orientation preserving subgroups of groups generated by reflections in certain hyperbolic pentahedra.

2.2. The case $p=4,5$ and the axis is simple. If there is a simple elliptic of order 4 or 5 , then the collaring theorems above are sufficient to the purpose once one knows the spectrum of axial distances sufficiently far out. 
This is because small axial distance implies the existence of icosahedral, octahedral or tetrahedral points. For $p=3$ finding these axial distances is exceedingly complicated and our estimates are not yet sufficient to the task.

2.3. The icosahedral, octahedral and tetrahedral cases. If there are octahedral or tetrahedral points, then the precisely invariant ball, whose construction is noted in Section 1.4, and the usual ball packing argument ([Me] and [GM2]) give a big enough volume, or imply the existence of an icosahedral point, except in the extremal case when $p=4$. However we have enough geometric information in this last case to eliminate it; indeed, we can actually construct the group. In the extremal case of an icosahedral subgroup, the precisely invariant ball and the ball packing trick give a very good lower bound, nearly equal to the conjectured minimal covolume. Calculating the next few icosahedral distances one obtains a bound larger than the conjectured extremal. Examining the extremal case we find that the closest two icosahedral points generate the index two subgroup of the group with Coxeter diagram 3-5-3. The two icosahedral points are conjugate, for otherwise we could double our volume estimate. We have therefore found that any group with co-volume smaller than that of $\Gamma_{353}$ contains $\Gamma_{353}$. Since both groups are co-finite volume, the index of one in the other is a finite integer. Our precisely invariant ball, and the packing argument, have shown us that this integer is less than 2, completing the proof.

In recent joint work with Alan Reid [GMR] we give a sufficient criterion for determining when a two generator Kleinian group with elliptic generators embeds in an arithmetic Kleinian group. It turns out that many of the extremal examples we need to consider are arithmetic. Indeed all the Kleinian groups with small axial distance are arithmetic in the case of an order three generator. Similar results holds for order four, five and six generators. This fact significantly simplifies the part of our analysis concerning these extremal groups.

\section{References}

[A1] C. C. Adams, The noncompact hyperbolic 3-manifold of minimal volume, Proc. Amer. Math. Soc. 100 (1987), 601-606.

[A2] L Limit volumes of hyperbolic three-orbifolds, J. Diff. Geom. 34 (1991), $115-141$.

[B] A. Beardon, The geometry of discrete groups, Springer-Verlag, 1983.

[CF] T. Chinburg and E. Friedman, The smallest arithmetic hyperbolic three-orbifold, Invent. Math. 86 (1986), 507-527.

[CM] M. D. E. Conder and G. J. Martin, Cusps, triangle groups and hyperbolic 3folds, Australian J. Math. (to appear). 
[CS1] M. Culler and P. B. Shalen, Paradoxical decompositions, 2-generator Kleinian groups, and volumes of hyperbolic 3-manifolds, J. Amer. Math. Soc. 5 (1992), $231-288$

[CS2] Volumes of hyperbolic Haken manifolds, I (to appear).

[DM] D. A. Derevnin and A. D. Mednykh, Geometric properties of discrete groups acting with fixed points in Lobachevsky space, Soviet Math. Dokl. 37 (1988), 614-617.

[GM1] F. W. Gehring and G. J. Martin, Iteration theory and inequalities for Kleinian groups, Bull. Amer. Math. Soc. 21 (1989), 57-63.

[GM2] _ Inequalities for Möbius transformations and discrete groups, J. reine angew. Math. 418 (1991), 31-76.

[GM3] _ Axial distances in discrete Möbius groups, Proc. Natl. Acad. Sci. USA 89 (1992), 1999-2001.

[GM4] , 6-torsion and hyperbolic volume, Proc. Amer. Math. Soc. 117 (1993), $727-735$.

[GM5] Commutators, collars and the geometry of Möbius groups, J. d'Analyse Math. 63 (1994) (to appear).

[GM6] - Torsion and volume in hyperbolic 3-folds (to appear).

[GM7] , Tetrahedral, octahedral and icosahedral subgroups of a Kleinian group (to appear).

[GM8] Commutator spectra for discrete groups with an elliptic generator (to appear).

[GMR] F. W. Gehring, G. J. Martin, and A. W. Reid,, Arithmeticity, discreteness and volume (to appear).

[H] A. Hurwitz, Über algebraische Gebilde mit eindeutigen Transformationen in sich, Math. Ann. 41 (1892), 403-442.

[KM] D. A. Kazdan and G. A. Margulis, A proof of Selberg's conjecture, Math. USSR, Sb. 4 (1968), 147-152.

$[\mathrm{KoM}]$ S. Kojima and Y. Miyamoto, The smallest hyperbolic 3-manifolds with totally geodesic boundary, J. Diff. Geom. 34 (1991), 175-192.

[M] B. Maskit, Kleinian groups, Springer-Verlag, 1987.

[Me] R. Meyerhoff, The cusped hyperbolic 3-orbifold of minimal volume, Bull. Amer. Math. Soc. 13 (1985), 154-156.

[T] W. P. Thurston, The geometry and topology of three-manifolds, Princeton University Lecture Notes, 1976.

[W] J. Weeks, Personal communication.

The Univerity of M ichigan, Ann Arbor, M I 48109 , U.S.A.

The Univerity of Auckland, Auckland, New Zealand and Centre For Mathematics \& Applications, Australian National Univerity, Canberra A C T, A ustralia

E-mail address: fgehring@math.lsa.umich.edu and martin@mat.auckland.ac.nz 